\title{
Status of Worker Exposure to Asphalt Paving Fumes With the Use of Engineering Controls
}

Supporting Information

\section{A. Data Handling}

In computation of geometric and arithmetic means, it was possible to obtain actual weights of samples less than the limit of detection (LOD), which were converted to concentration measurements just as was done for samples greater than the limit of detection. Since these values were used in averages, this should not represent a problem for data reliability.

For the worker and area data, confidence limits were required for both geometric means and TWAs, and because the variance of measurements increased with the concentration, all confidence intervals were computed on the natural $\log$ scale. A problem is how to deal with negative concentrations. Averaging simultaneous samples before taking logs was a way to deal with negative values, since for most of the paired concentrations that were averaged, no more than one concentration was negative. Averaging made many of these values usable for the worker data, but since the area samples were not collected in simultaneous pairs, no averaging could be done for those samples. The sample locations that still had negative results after application of this procedure were: for both TPM and BSM one worker sample at Site 1; for BSM, two worker samples and one area sample at Site 2; for BSM, two area samples at Site 3. There is no reason to automatically discredit negative data, since none of the TPM concentrations were less than the BSM concentrations within the negative data set. Since geometric means and TWAs were required for each site, as well as confidence limits for these averages, 
it seemed best to provide substitute values, so as not to discard data, unless they were possible outliers. When the average of the two samples for a worker on the given day was negative or when the single area sample was negative, the substitute value was chosen as the minimum of the following two selections: a) for the worker samples, if one of the two samples was positive, select that sample; b) if both worker samples were negative or if the single area sample was negative, select the average of the positive background samples taken at the site for the two days. The choice of the minimum of selections a) and b) provided substitute values for all missing values. In addition, there were three sites, at which sampling results for four locations (three for TPM and one for BSM) for the two days were different enough to appear as outliers, though the results were positive. Rather than delete both days' data, the two results were averaged and the average was used in the calculations.

\section{B. Statistical Models; Confidence Intervals for TWAs}

Two statistical models were used to produce confidence intervals for the estimates for the TPM and BSM worker and area data. The first kind of model was required to provide confidence intervals for the TPM and BSM geometric means given in the paper. The data used were the observed concentrations for each sample (not adjusted to 480 minutes, as was done for the TWA results). Sites, days at sites, and residual error were treated as random samples, and means were estimated by averaging over paving manufacturer and sample location. (The confidence intervals from this kind of model are those given in the abstract.) For the second kind of model, a site mean was estimated for worker and area TWA samples for both BSM and TPM. In these models, days at site were treated as random, in addition to the residual error. (The confidence limits from the second model appear in Table 1.)

Whereas the confidence limits for geometric means in the first kind of model are obtained by exponentiating the ln-scale confidence limits, those for TWAs in the second kind of model use Waldtype limits $(1,2)$. The problem of interest in the references $(1,2)$ also concerns confidence intervals for an arithmetic average, when the data are lognormal, but the designs from which the data come are 
somewhat different from the data considered here. To deal with cross-classified data and with multiple variance components on the log scale, the following approach was taken. The form of the Wald-type confidence interval for an approximation to the true arithmetic mean is as follows, where $\mathrm{x}=[$ sample mean $(\ln (\mathrm{y}))+$ sample variance/2]:

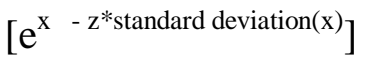

$$
\begin{aligned}
& <\operatorname{arithmetic~mean~}<\left[\mathrm{e}^{\mathrm{x}+\mathrm{z}^{*} \text { standard deviation }(\mathrm{x})}\right]
\end{aligned}
$$

where $\mathrm{z}$ is the appropriate quantile of the normal distribution required to obtain, for example, a $95 \%$ confidence statement. Since the Wald confidence intervals are too narrow for small and moderate-sized samples, we follow the suggestion (2) that the $\mathrm{z}$ value be used that corresponds to confidence level (1$\alpha / 2)$ when $(1-\alpha)$ is desired, if there are two components of variance, and the non-residual component (between day variance for this data) is at least half the residual component, which is true here. Since 95\% confidence intervals are desired, instead of using 1.96 for z, 2.24 is used (since the probability that a normal variable will exceed 2.24 is 0.0125 , and $2(0.0125)$ is $0.025=\alpha$ for each side of the two-sided confidence interval.) When there are multiple variance components, the variance required can be computed as follows:

$\operatorname{Var}[$ sample mean +0.5 (sample variance $)]=$

$\operatorname{Var}[$ sample mean] $+0.25 \Sigma$ Var[estimated var comp est(i)] $+0.25 \Sigma$ (over all $\mathrm{i} \neq \mathrm{j}) \operatorname{cov[estimated}$ $\operatorname{var}(\mathrm{i})$, estimated $\operatorname{var}(\mathrm{j})]$,

where the estimated variances and covariances used in the work presented are the asymptotic variances and covariances produced by Proc Mixed in SAS (3), and where sample mean and variance component estimates are approximately uncorrelated for balanced data. Some simulations were done to study the probability content of this confidence interval. These results indicate that even with the $\alpha / 2$ correction, the confidence intervals are still too narrow. However, because of the limited amount of 
simulation work carried out, we are reluctant to propose a better correction for the Wald interval.

\section{Confidence Intervals for 15-Minute Sample Medians}

The 15-minute samples had negative values, and it seemed sensible to use medians. In addition, the median for a lognormal distribution is identical to the geometric mean. For the eight 15-minute samples at each site, the median was computed for both TPM and BSM. For calculation of confidence limits, Site 12 was excluded. The remaining eleven medians were treated as a random sample, and $95 \%$ confidence intervals were constructed for TPM and BSM by means of the ordinary sign test, whereby, for eleven values, the second smallest and second largest provide the 95\% confidence limits (4).

\section{Confidence Intervals for Geometric Count Mean Diameters}

The confidence intervals shown in Figure 2 were computed as follows. The original data were not available to the authors. It was known that the data were collected in sets of three consecutive twentysecond determinations. For most days there were two of these sets of measurements made for each worker, one in the morning and one in the afternoon. Thus, for the three workers there were six sets of these measurements per day, and twelve per site (Site 4 had just one day of data, or six sets.) A conservative approach is to assume that all variability is between sets. Under this assumption, we converted the standard deviation estimates based on 12(3)-1 degrees of freedom to those based on eleven degrees of freedom, assuming that there was no variation within each set. The confidence statements were then based on these recomputed standard deviations, with eleven degrees of freedom. (For Site 4 the recomputed standard deviations had five degrees of freedom.) Confidence limits were computed for the means on the natural log scale, using the recomputed standard deviations and the Student's t-distribution, and the limits were exponentiated to get the confidence limits for the geometric means on the original scale.

(1) Lyles, R.H. and L.L. Kupper: On Strategies for Comparing Occupational Exposure Data to 
Limits. American Industrial Hygiene Association Journal, 57: 6-15, 1996.

(2) Lyles, R.H., L.L. Kupper, and S.M. Rappaport: Assessing Regulatory Compliance of Occupational Exposures via the Balanced One-Way Random Effects ANOVA Model. Journal of Agricultural, Biological, and Environmental Statistics, 2: 64-86, 1997.

(3) SAS/STAT User's Guide, Version 8. SAS Institute, Inc. Cary, NC, 2083, 1999.

(4) Gibbons, J.D., Nonparametric Statistical Inference. McGraw-Hill, 104, 1971.

\section{E. Background Data}

The Figure below includes average TPM and BSM TWAs of the background samples by site as well as averages over all sites. The levels at Sites 2 and 9 are particularly alarming for background data, and appear to be related to the limits of the testing procedure.






\section{F. Geometric Count Mean Particle Size Data by Site}

Included in this section are two figures. The first figure shows the geometric count mean particle size data by site. Allowing real time exposure assessment, the second figure shows the particle size monitoring equipment (TSI Model 3320 Aerodynamic Particle Sizer ${ }^{\circledR}$ Spectrometer) in use by the industrial hygienist, Adam Redman, on top of a paver unit.
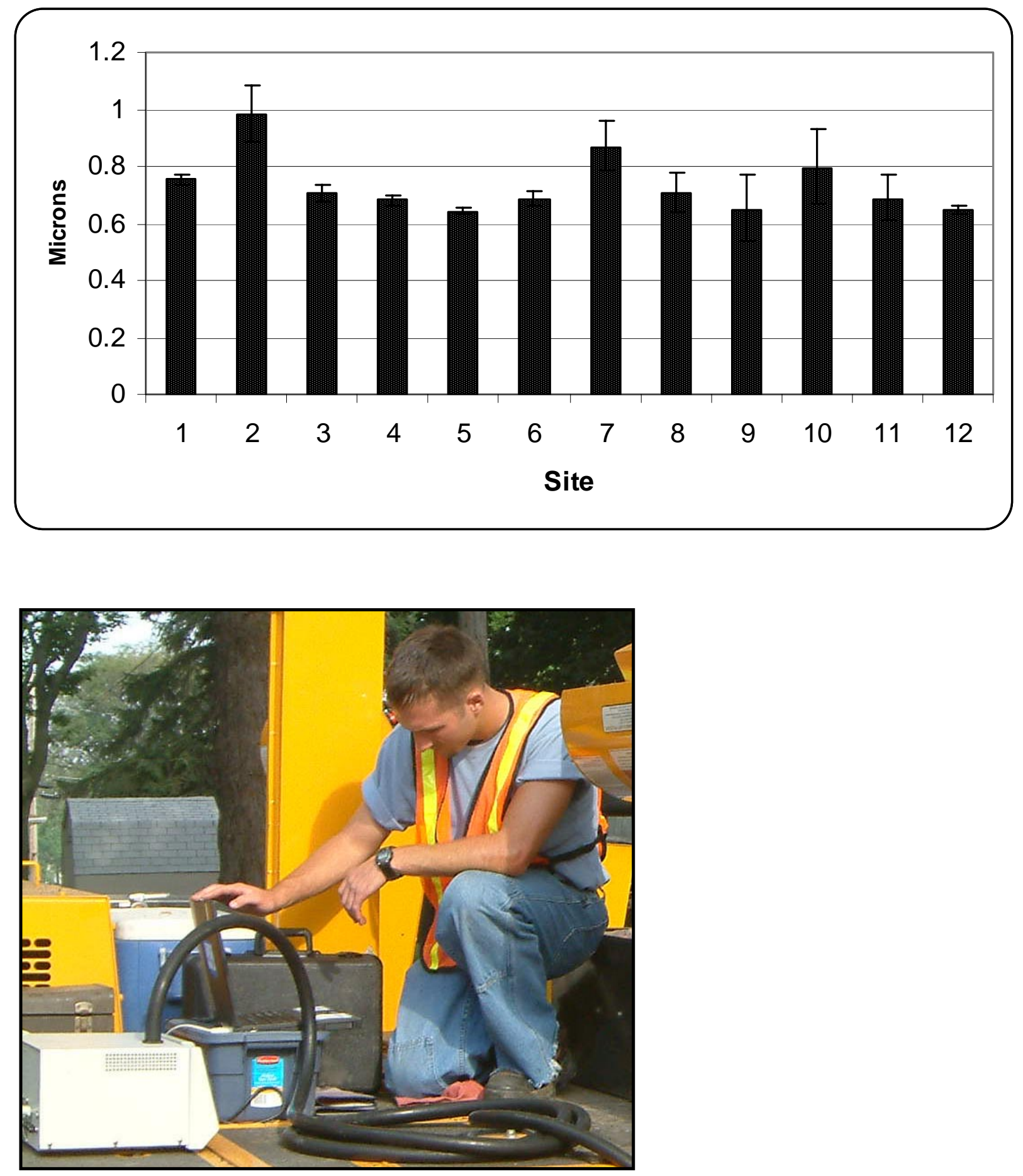